\title{
Response models and minimal designs for mixtures of $n$ of $m$ items useful for intercropping and other investigations
}

BY W. T. FEDERER

Biometrics Unit, Cornell University, Ithaca, NY I4853, U.S.A.

\author{
AND D. RAGHAVARAO \\ Department of Statistics, Temple University, Philadelphia, PA 19122, U.S.A. \\ BU-890-M-B \\ October 1986
}

\section{SUMMARY}

Consider the situation where $n$ of $m$ cultivars are grown together in a mixture such as that found in intercropping investigations. Response model equations for $n=2$ are formulated in a manner akin to that found for a diallel crossing experiment in genetics. This situation varies considerably from diallel crossing in that $n \geq 2$ and yields may or may not be available for each member of the mixture. Response model equations were formulated and then minimal treatment designs were obtained to derive least squares solutions for the parameters of the model. This was done for both cases, i.e., when individual member yields were avallable and when they were not. Variances of estimable contrasts are also given. Applications to other areas of investigation with examples are given.

Some key words: Balanced incomplete block; N-blends; General mixing ability; Specific mixing ability.

* In the Technical Report Series of the Biometrics Unit, Cornell University. 


\section{INTRODUCTION}

Intercropping investigations involve the growing of two or more cultivars on the same area of land, where a cultivar may be a line, variety, and/or species. It is a centuries-old practice in tropical agriculture, and to some extent in temperate zone agriculture. Agricultural, biological, and statistical investigations have tended to ignore the problems of research in this area due to the complexities involved in modeling and interpreting such experiments.

Consider an investigation involving m cultivars, say $\{1, \cdots, m\}$. One can form $v$ non-empty sets $s_{1}, \cdots, s_{v}$ of the $m$ cultivars. These sets may all be of equal or unequal sizes and they may be of any cardinality $1,2, \cdots, m$. The class of sets so formed is called the treatment design of the $m$ cultivars and these $v$ treatments will be used in an appropriate experiment design like a completely randomized design, a randomized complete block design, an incomplete block design, etc. If the set $s_{\alpha}$ has a single element $i$, then $s_{\alpha}=\{i\}$ is called the $i^{\text {th }}$ sole cultivar or uni-blend. If $s_{\alpha}=\{i, j\}$, then the treatment $s_{\alpha}$ is called the bi-blend of cultivars $i$ and j. If $s_{\alpha}=\{i, j, k\}$ then the treatment $s_{\alpha}$ is called the tri-blend of cultivars $1, j$, and $k$. If $s_{\alpha}=\left\{1_{1}, \cdots, 1_{n}\right\}$, the treatment $s_{\alpha}$ is then called the $n$-blend of cultivars $1_{1}, \cdots, 1_{n}$.

For simplicity, let us consider the experiment design used with the $v$ treatments to be an orthogonal design. The data can be collected either for each Individual cultivar of the treatment $s_{\alpha}$ associated with an experimental unit, or the data can be collected for the whole experimental unit to which the treatment $s_{\alpha}$ is applied. Let $s_{\alpha}=\left\{i_{1}, \cdots, i_{n}\right\}$. In the former case, let $\bar{y}_{i_{j}}\left(s_{\alpha}\right)$ denote the mean yield of the $i_{j}$ cultivar used in the treatment $s_{\alpha}$ for $j=1, \cdots, n$. In the latter case, let $\bar{y}_{s_{\alpha}}$ denote the mean 
yield of the treatment $\mathrm{s}_{\alpha}$. Note that in $\bar{y}_{S_{\alpha}}$ only the total of $n$ cultivars is available and the individual cultivar yields are not. If more complicated experiment designs are used for the $v$ treatments, one may replace $\bar{y}_{i_{j}}\left(s_{\alpha}\right)$ and $\bar{y}_{S_{\alpha}}$ by the estimated adjusted treatment effects appropriate to the experiment design used. Thus without loss of generality we assume the response variable $\bar{y}_{i_{j}}\left(s_{\alpha}\right)$ or $\bar{y}_{S_{\alpha}}$ for further analyses discussed in this paper.

In Section 2 we develop a model to interpret $\bar{y}_{i_{j}}\left(s_{\alpha}\right)$ and give minimal designs which will enable us to estimate all the parametric contrasts of interest. In Section 3, an analysis of such minimal designs will be presented. In Sections 4 and 5 a similar approach will be used for $\bar{y}_{S_{\alpha}}$. In the concluding section we will give some applications.

2. MODEL AND MINIMAL DESIGNS WITH RESPONSE VARIABLE $\bar{y}_{i_{j}}\left(S_{\alpha}\right)$ When we consider the response of a cultivar $i_{j}$ used in the treatment $s_{\alpha}=\left\{i_{1}, \cdots, i_{n}\right\}$, it may be affected by the following components:

(i) its relative performance as a monoculture or uni-blend, and such an effect will be denoted by $\mu+\tau_{i}^{*}$;

(ii) Its effect because of its use in mixtures, and such an effect will be called general mixing ability. It will be denoted by $\delta_{i_{j}}$;

(iii) its ability to respond well (or poorly) because of the presence of each of the other n-1 cultivars in that blend. We denote such effects by $\gamma_{i_{j}}\left(i_{k}\right)$ for $k=1, \cdots, n ; k \neq j$ and we call them the first order specific mixing abilities of the $i_{j}$ cultivar with the $i_{k}$ cultivar. One can easily note that $\gamma_{i_{j}}\left(1_{k}\right) \neq \gamma_{i_{k}}\left(i_{j}\right)$ and thus the first order mixing abilities are not the usual first order interaction of the cultivars $i_{j}$ and $i_{k}$; 
(iv) its ability to respond well (or poorly) because of the presence of each distinct pair of the other $n-1$ cultivars in that blend. We denote such effects by $\gamma_{i_{j}}\left(i_{k}, i_{\ell}\right)$ for $k, l=1, \cdots, n ; k \neq j, l \neq j, k<l$, and they can be called second order specific mixing abilities of the $i_{j}$ cultivar with $i_{k}$ and $i_{\ell}$ cultivars; and

(v) Continuing in this fashion, it depends on the third, $\cdots,(n-1)^{\text {th }}$ order specific mixing abilities of the $i_{j}$ cultivar with the other cultivars.

As an illustration, it can be noted that

$$
\begin{gathered}
E\left(\bar{y}_{i_{1}\left(s_{\alpha}\right)}\right)=\mu+\tau_{i_{1}}^{*}+\delta_{i_{1}}+\sum_{k=2}^{n} \gamma_{i_{1}\left(i_{k}\right)}+\sum_{k, \ell ; k<\ell ; k, l \neq 1}^{n} \gamma_{i_{1}}\left(i_{k}, i_{\ell}\right) \\
+\cdots+\gamma_{i_{1}\left(i_{2}, \cdots, i_{n}\right),}
\end{gathered}
$$

where $\mathrm{E}(\cdot)$ is the expected value of the random variable in the parentheses.

In an experiment involving $m$ cultivars, it is possible for each cultivar to have a monoculture effect, general mixing ability, specific mixing abilities of first, $\cdots$, and $(m-1)^{\text {th }}$ order. In practice, an experimenter may be interested in drawing inferences on at most $t^{\text {th }}$ order specific mixing abilities for a given $t(t \leq m-1)$. By using uni-blends, bi-blends, - . , $(t+1)$-blends, one can make such inferences. When using mixtures with different number of cultivars, the problems of plant density per hectare for each cultivar in the mixture and of unequal error variances arise. It becomes absolutely necessary to use uni-blends if the experimenter is interested in drawing conclusions about general mixing abilities. If uni-blends are not used in the experiment, $\tau_{i j}^{*}$ and $\delta_{i j}$ effects will be completely confounded, and one can draw inferences only about $\tau_{i j}=\tau_{i j}^{*}+\delta_{i j} \cdot$ In this paper, we restrict our attention to 
blends using the same number of cultivars, that is, the treatment sets $s_{\alpha}$ having the same cardinality.

The effects can be reparameterized, if necessary, and the following conditions or restrictions may be imposed on the parameters:

$$
\begin{aligned}
& \sum_{i=1}^{m} \tau_{i}=0 \\
& \sum_{j_{1}=1}^{m} \gamma_{i\left(j_{1}\right)}=0, \text { for a given } i, j_{1} \neq i \\
& \sum_{j_{2}=1}^{m} \gamma_{i\left(j_{1}, j_{2}\right)}=0, \text { for a given } i \text { and } j_{1}\left(i \neq j_{1}\right), j_{2} \neq i, j_{1} \\
& \vdots \\
& \sum_{j_{t}=1}^{m} r_{i\left(j_{1}, \cdots, j_{t}\right)}=0 \text {, for a given } i \text { and } j_{1}, \cdots, j_{t-1} \text {, } \\
& \left(j_{1}<j_{2}<\cdots<j_{t-1}, i \neq j_{1}, \cdots, j_{t-1}\right), j_{t} \neq i, j_{1}, \cdots, j_{t-1}
\end{aligned}
$$

For any $k$, the number of $k^{\text {th }}$ order specific combining ability parameters on the $i^{\text {th }}$ cultivar, $\gamma_{i\left(j_{1}, \cdots, j_{k}\right)}$ is $(m-1)$ choose $k$, and the number of restrictions is $(m-1)$ choose $(k-1)$. Thus, the number of independent $k^{\text {th }}$ order specific combining ability parameters on the $1^{\text {th }}$ cultivar is

$$
\left(\begin{array}{c}
m-1 \\
k
\end{array}\right)-\left(\begin{array}{c}
m-1 \\
k-1
\end{array}\right)=\frac{(m-1) !}{k !(m-k) !}(m-2 k)
$$

and hence the total number of $k^{\text {th }}$ order specific combining ability parameters is

$$
m \frac{(m-1) !}{k !(m-k) !}(m-2 k)=\left(\begin{array}{l}
m \\
k
\end{array}\right)(m-2 k)
$$


In view of this, to draw inferences about $\tau_{i}$ and at most $t^{\text {th }}$ order specific mixing abilities of the cultivars, the number of responses needed is

$1+(m-1)+\left(\begin{array}{c}m \\ 1\end{array}\right)(m-2)+\left(\begin{array}{c}m \\ 2\end{array}\right)(m-4)+\cdots+\left(\begin{array}{c}m \\ t\end{array}\right)(m-2 t)=\left(\begin{array}{c}m \\ t+1\end{array}\right)(t+1)$ for the mean, $\tau_{i} s$ (cultivar effects in a mixture), and first to $t^{\text {th }}$ order specific mixing abilities, respectively.

Thus using $m$ cultivars, one can draw inferences for at most $[\mathrm{m} / 2-1]^{\text {th }}$ order specific mixing abilities where [·] is the greatest integer function because $m-2 t>0$. On the other hand, if an experimenter is interested in estimating $t^{\text {th }}$ order specific mixing abilities, the number of cultivars to be used in the experiment $m$ should satisfy $m>2 t$. The required number $(t+1)$ times $m$ choose $(t+1)$ of responses can be obtained by using $v$ equal to $m$ choose $(t+1)$ treatment sets $s_{\alpha}$, where the sets $s_{1}, \cdots, s_{v}$ form an irreducible balanced incomplete block design of all combinations of $(t+1)$ cultivars selected from the $m$ cultivars and then noting the response on each of the $(t+1)$ cultivars in the mixture treatment sets $s_{\alpha}$. Such designs are minimal designs where a minimal treatment design is one in which the number of responses for the cultivars in mixtures is smallest for estimating the parametric contrasts of interest.

A solution of the normal equations for the unknown parameters for estimating the required contrasts can be easily obtained, and will be given for $t=2$ in the next section.

\section{A SOLUTION OF PARAMETERS FOR TREATMENT DESIGNS OF SECTION 2}

Consider an experiment with $m$ cultivars in which the experimenter is interested in drawing inferences up to second order specific mixing 
abilities. As noted in the last section, the minimal treatment design consists of $v$ equals $m$ choose 3 treatments $s_{1}, \cdots, s_{v}$ is made up of all triples of the m cultivars and obtaining responses for each of the 3 cultivars in each mixture. However, an experimenter may be interested in using blends of $n$ cultivars, $n \geq 3$. The treatments can be laid out in an appropriate experiment design. Let $\bar{y}_{i_{j}\left(s_{\alpha}\right)}$ be the mean response of the $i_{f}$ cultivar used in the mixture $s_{\alpha}$ from an orthogonal experiment design. One adjusts $\bar{y}_{i_{j}}\left(s_{\alpha}\right)$ for blocks if a non-orthogonal experiment design is used. We give the results here using $\bar{y}_{i_{j}}\left(s_{\alpha}\right)$ from an orthogonal experiment design for general $n$ and specialize the results for $n=3$.

As a consequence of the conditions or restrictions imposed on the parameters, the following is a solution for the parameters which can be used to estimate the contrasts of $\tau_{i} s, \gamma_{i(j)} s$, and $\gamma_{i(j, k)} s$ which are of interest to the experimenter:

$$
\begin{aligned}
\hat{\tau}_{i} & =\left(\begin{array}{l}
m-1 \\
n-1
\end{array}\right)^{-1} \sum_{\alpha ; i \varepsilon S_{\alpha}} \bar{y}_{i\left(s_{\alpha}\right)}-\tilde{y}, \\
\hat{r}_{i(j)} & =\left[(m-1)\left(\begin{array}{c}
m-3 \\
n-2
\end{array}\right)\right]^{-1}\left[\begin{array}{c}
\left.(m-n) \sum_{\alpha ; i, j \varepsilon S_{\alpha}} \bar{y}_{i\left(s_{\alpha}\right)}-(n-1) \sum_{\alpha ; i \varepsilon S_{\alpha}, j \notin S_{\alpha}} \bar{y}_{i(s}\right)
\end{array},\right.
\end{aligned}
$$

and

$$
\hat{\gamma}_{i(j k)}=\frac{1}{s}\left[\sum_{\alpha ; i, j, k \varepsilon S_{\alpha}} \bar{y}_{i\left(s_{\alpha}\right)}-\left(\begin{array}{c}
m-3 \\
n-3
\end{array}\right)\left(\tilde{y}+\hat{\tau}_{i}\right)-\left(\begin{array}{l}
m-4 \\
n-3
\end{array}\right)\left\{\hat{\gamma}_{i(j)}+\hat{\gamma}_{i(k}\right\}\right],
$$

where

$$
\tilde{y}=\sum_{i, \alpha} \bar{y}_{i\left(s_{\alpha}\right)} /\left\{\left(\begin{array}{l}
m \\
n
\end{array}\right) n\right\}
$$

and

$$
s=\left(\begin{array}{l}
m-3 \\
n-3
\end{array}\right)-2\left(\begin{array}{l}
m-4 \\
n-4
\end{array}\right)+\left(\begin{array}{l}
m-5 \\
n-5
\end{array}\right)
$$


When $n=3$ the above simplify to:

$$
\begin{aligned}
\hat{\tau}_{i} & =\left(\begin{array}{c}
m-1 \\
2
\end{array}\right)^{-1} \sum_{\alpha ; i \varepsilon S_{\alpha}} \bar{y}_{i\left(s_{\alpha}\right)}-\tilde{y}, \\
\hat{\gamma}_{i(j)} & =\frac{1}{(m-1)(m-3)}\left[(m-3) \sum_{\alpha ; i, j \in S_{\alpha}} \bar{y}_{i\left(s_{\alpha}\right)}-2 \alpha_{\alpha ; i \varepsilon S_{\alpha}, j \notin S_{\alpha}} \bar{y}_{i\left(s_{\alpha}\right)}\right]
\end{aligned}
$$

and

$$
\begin{aligned}
\hat{\gamma}_{i(j, k)=} & \frac{1}{(m-2)(m-3)}\left[(m-3)(m-4) \sum_{\alpha ; i, j, k \in S_{\alpha}} \bar{y}_{i\left(s_{\alpha}\right)}\right. \\
& -(m-4) \sum_{\alpha ; i, j \varepsilon S_{\alpha} ; k \notin s_{\alpha}} \bar{y}_{i\left(s_{\alpha}\right)-(m-4)} \sum_{\alpha ; i, k \varepsilon S_{\alpha} ; j \notin S_{\alpha}} \bar{y}_{i\left(s_{\alpha}\right)} \\
& \left.+2 \sum_{\alpha ; i \varepsilon S_{\alpha} ; j, k \notin S_{\alpha}} \bar{y}_{1\left(s_{\alpha}\right)}\right]
\end{aligned}
$$

where

$$
\tilde{y}=\sum_{i, \alpha} \bar{y}_{i\left(s_{\alpha}\right)},\left\{\left(\begin{array}{c}
m \\
3
\end{array}\right) 3\right\}
$$

is the overall mean of the means.

If the means of the cultivar blends are based on $r$ replications and if $\sigma^{2}$ is the error variance for the responses in the original orthogonal design

$$
\begin{aligned}
\operatorname{var}\left(\hat{\tau}_{i}-\hat{\tau}_{i^{\prime}}\right)=2 \sigma^{2} /\left\{\left(\begin{array}{c}
m-1 \\
2
\end{array}\right) r\right\}, & i \neq i^{\prime}, \\
\operatorname{var}\left(\hat{\gamma}_{i(j)}-\hat{\gamma}_{i\left(j^{\prime}\right)}\right)=2 \sigma^{2} /\{(m-3) r\}, & j \neq j^{\prime}, \\
\operatorname{var}\left(\hat{\gamma}_{i(j, k)}-\hat{\gamma}_{i\left(j, k^{\prime}\right)}\right)=2(m-4) \sigma^{2} /\{(m-3) r\}, & k \neq k^{\prime},
\end{aligned}
$$

and

$$
\operatorname{var}\left(\hat{\gamma}_{i(j k)}-\hat{\gamma}_{i\left(j^{\prime} k^{\prime}\right)}\right)=2(m-5) \sigma^{2} /\{r(m-3)\}, \quad(j, k) \neq\left(j^{\prime}, k^{j}\right)
$$


Expressions for the solutions of parameters can be similarly obtained for other designs of this type, when the interest is in drawing inferences about higher order specific mixing abilities. An analysis of variance can be obtained by standard methods.

4. MODEL AND MINIMAL DESIGNS WITH RESPONSE VARIABLE $\bar{y}_{S_{\alpha}}$

Subject to the terminology introduced in section 1 , if $\bar{y}_{S}$ is the mean of the mixture treatment $s_{\alpha}=\left\{i_{1}, \cdots, i_{n}\right\}$ based on the orthogonal experiment design used,

$$
\begin{aligned}
E\left(\vec{y}_{S_{\alpha}}\right)=n \mu & +\sum_{j=1}^{n} \tau_{i_{j}}^{*}+\sum_{j=1}^{n} \delta_{i}^{*}+\sum_{j, k=1 ; j \neq k}^{*} \gamma_{i_{j}\left(t_{k}\right)}+\sum_{j, k, l=1 ; j \neq k \neq l \neq j} \sum_{i_{j}\left(i_{k}, i_{l}\right)}^{n} \\
& +\cdots+\gamma_{i_{1}\left(i_{2}, \cdots, i_{n}\right)}+\gamma_{i_{2}\left(i_{1}, \cdots, i_{n}\right)}+\cdots+\gamma_{i_{n}}\left(i_{1}, \cdots, i_{n-1}\right) \\
=\mu^{*}+ & \sum_{j=1}^{n} \tau_{i_{j}}+\sum_{j, k=1 ; j<k}^{n} \lambda_{i_{j} i_{k}}+\sum_{j, k, l=1 ; j<k<l} \lambda_{j} i_{k} i_{l}+\cdots+\lambda_{i}, \cdots, i_{n},
\end{aligned}
$$

where

$$
\begin{aligned}
& \lambda_{i_{j} i_{k}}=\gamma_{i_{j}\left(i_{k}\right)}+\gamma_{i_{k}\left(i_{j}\right)}, \lambda_{i_{j} i_{k} i_{l}}=\gamma_{i_{j}}\left(i_{k}, i_{l}\right) \\
& +\gamma_{i_{k}\left(i_{j}, i_{\ell}\right)}+\gamma_{i_{\ell}\left(i_{j}, i_{k}\right)}, \cdots \lambda_{i_{1}}, \cdots, i_{n}=\gamma_{i_{1}}\left(i_{2}, \cdots, i_{n}\right) \\
& +\gamma_{i_{2}}\left(i_{1}, \cdots, i_{n}\right)+\cdots \gamma_{i_{n}}\left(i_{1}, \cdots, i_{n-1}\right) .
\end{aligned}
$$

For convenience, we may put $\lambda_{i_{k} i_{j}}=\lambda_{i_{j} i_{k}}, \lambda_{i_{j} i_{k} i_{\ell}}=\lambda_{i_{j}{ }^{1} l_{k}}=\lambda_{i_{k}{ }^{i}{ }^{i} \ell}$ $=\lambda_{i_{k} i_{l} i_{j}}=\lambda_{i_{l}} i_{j} i_{k}=\lambda_{i_{l} i_{k} i_{j}}$, etc. The parameter $\lambda_{i_{j} i_{k}}$ behaves like an interaction term for the $1_{j}$ and $i_{k}$ cultivars; but in essence it is the sum of combining abilities of $1_{j}$ cultivar with $i_{k}$ and that of $1_{k}$ cultivar with $i_{j}$ A similar interpretation can be given for the other $\lambda$ parameters. In 
the same vein as in Section 2 , we may call $\lambda_{i_{j} i_{k}}$ as the first order specific mixing ability of $i_{j}$ and $i_{k}$ cultivars, $\lambda_{i_{j}} i_{k}{ }^{1}$ as the second order specific mixing ability of $i_{j}, i_{k}$ and $i_{\ell}$ cultivars, $\cdots, \lambda_{1_{1}}, \cdots, i_{n}$ as the $n^{\text {th }}$ order specific mixing ability of $i_{1}, \cdots, i_{n}$ cultivars.

As in Section 2, the effects can be reparameterized, if necessary, and the following conditions or restrictions may be imposed on the parameters:

$$
\begin{aligned}
& \sum_{i=1}^{m} \tau_{i}=0, \\
& \sum_{i=1}^{m} \lambda_{i} i_{k}=0 \text {, for a given } i_{k} \neq i_{j} \text {, } \\
& \sum_{i_{k}=1}^{m} \lambda_{i_{j} i_{k}}=0 \text {, for a given } i_{j} \neq i_{k} \text {, } \\
& \sum_{i_{j}=1}^{m} \lambda_{i_{j} i_{k} i_{\ell}}=0 \text {, for given } i_{k} \text { and } i_{\ell}, i_{k} \neq i_{\ell}, i_{j} \neq i_{k}, i_{j} \neq i_{\ell} \text {, } \\
& \sum_{i_{k}=1}^{m} \lambda_{i_{j} i_{k} i_{l}}=0 \text {, for given } i_{j} \text { and } i_{l}, i_{j} \neq i_{l}, i_{k} \neq i_{j}, i_{k} \neq i_{l} \\
& \sum_{i_{l}=1}^{m} \lambda_{i_{j} i_{k} i_{l}}=0 \text {, for given } i_{j} \text { and } i_{k}, i_{j} \neq i_{k}, i_{\ell} \neq i_{j}, i_{l} \neq i_{k}, \\
& \sum_{i_{1}=1}^{m} \lambda_{i_{1}}, \cdots, i_{n}=0, i_{1} \neq i_{2}, \cdots, i_{n}, \quad \sum_{i_{2}=1}^{m} \lambda_{i_{1}}, \cdots, i_{n}=0, i_{2} \neq i_{1}, \cdots, i_{n} \\
& \cdots \quad \sum_{i_{n}=1}^{m} \lambda_{i_{1}}, \cdots, i_{n}=0, i_{n} \neq i_{1}, i_{2}, \cdots, i_{n-1} .
\end{aligned}
$$

For any $k$, the number of $k^{\text {th }}$ order specific combining ability parameters $\lambda_{i_{1}}, \cdots, i_{k+1}$ is $m$ choose $(k+1)$ and the number of restrictions imposed 
is $m$ choose $k$. Thus the number of independent $k^{\text {th }}$ order specific combining ability parameters is

$$
\left(\begin{array}{c}
m \\
k+1
\end{array}\right)-\left(\begin{array}{l}
m \\
k
\end{array}\right)=\left(\begin{array}{l}
m \\
k
\end{array}\right) \frac{(m-2 k-1)}{(k+1)}
$$

If the experimenter is interested in drawing inferences about the $\tau_{i}$ and at most $t^{\text {th }}$ order specific mixing abilities of the cultivars using mixtures of the same size with one response for each mixture, the number of responses needed is

$$
1+(m-1)+\left(\begin{array}{l}
m \\
1
\end{array}\right) \frac{(m-3)}{2}+\left(\begin{array}{l}
m \\
2
\end{array}\right) \frac{(m-5)}{3}+\cdots+\left(\begin{array}{c}
m \\
t
\end{array}\right) \frac{(m-2 t-1)}{t+1}=\left(\begin{array}{c}
m \\
t+1
\end{array}\right) .
$$

Thus using $m$ cultivars one can draw inferences on at most $[(m-1) / 2]^{\text {th }}$ order specific mixing abilities. On the other hand, if an experimenter is interested in estimating $t^{\text {th }}$ order specific mixing abilities, the number of cultivars to be used in the experiment $m$ should satisfy $m>2 t+1$. Note that in Section $2, m>2 t$, whereas here we need $m>2 t+1$. The required number $m$ choose $(t+1)$ of responses can be obtained by using $v$ equals $m$ choose $(t+1)$ treatments $s_{\alpha}$, where the sets $s_{1}, \cdots, s_{v}$ form an irreducible balanced imcomplete block design of all combinations of $(t+1)$ cultivars selected from the $m$ cultivars and then noting the response on each of the $v$ treatments $\mathrm{S}_{\alpha}$. Such designs are minimal designs for estimating all the parametric contrasts of interest. A solution of the normal equations for the unknown parameters to estimate the required contrasts can be easily obtained and will be given for $t=2$ in the next section.

\section{A SOLUTION FOR PARAMETERS FOR THE TREATMENT DESIGNS OF SECTION 4}

Consider an experiment with $m$ cultivars on which the experimenter is interested in drawing inferences up to second order specific mixing abilities as noted in Section 4; the treatment design consists of $v$ equals 
$m(m-1)(m-2) / 6$ treatments $s_{1}, \cdots, s_{v}$ consisting of all triples of the $m$ cultivars and noting the responses on the treatment mixtures. The treatments can be laid out in an appropriate experiment design. Let $\bar{y}_{S_{\alpha}}$ be the mean response of the $S_{\alpha}$ treatment mixture when the experiment design is an orthogonal one. One may replace $\bar{y}_{S_{\alpha}}$ by adjusting it for block effects if a non-orthogonal experiment design is used. We give the results here using $\overline{\mathrm{y}}_{\mathrm{S}}$.

It can be easily verffied that the following is a solution of the parameters which can be used to estimate the contrasts of interest of $\tau_{i} s$, $\lambda_{i j} s$, and $\lambda_{i j k} s$, when $n(\geq 3)$ cultivars are used in each $s_{\alpha}$ consisting of all n-tuples of the $m$ cultivars:

$$
\begin{aligned}
& \widehat{\tau}_{i}=\left\{\sum_{\alpha ; i \varepsilon S_{\alpha}} \vec{y}_{S_{\alpha}}-\left(\begin{array}{c}
m-1 \\
n-1
\end{array}\right) \tilde{y}\right\} /\left(\begin{array}{c}
m-2 \\
n-1
\end{array}\right), \\
& \hat{\lambda}_{i j}=\left\{\sum_{\alpha ; i, j \in S_{\alpha}} \bar{y}_{S_{\alpha}}-\left(\begin{array}{l}
m-2 \\
n-2
\end{array}\right) \tilde{y}-\left(\begin{array}{l}
m-3 \\
n-2
\end{array}\right)\left(\hat{\tau}_{i}+\hat{\tau}_{j}\right)\right\} / w, \\
& \hat{\lambda}_{i j k}=\left\{\sum_{\alpha ; i, j, k \varepsilon S_{\alpha}} \bar{y}_{\alpha}-\left(\begin{array}{c}
m-3 \\
n-3
\end{array}\right) \tilde{y}-\left(\begin{array}{c}
m-4 \\
n-3
\end{array}\right)\left(\hat{\tau}_{i}+\hat{\tau}_{j}+\hat{\tau}_{k}\right)-s\left(\hat{\lambda}_{i j}+\hat{\lambda}_{i k}+\hat{\lambda}_{j k}\right)\right\} / u,
\end{aligned}
$$

where $s$ is given previously,

$$
\begin{aligned}
& \tilde{y}=\sum_{\alpha}^{-} s_{\alpha} /\left(\begin{array}{c}
m \\
n
\end{array}\right), \\
& w=\left(\begin{array}{l}
m-2 \\
n-2
\end{array}\right)-2\left(\begin{array}{l}
m-3 \\
n-3
\end{array}\right)+\left(\begin{array}{c}
m-4 \\
n-4
\end{array}\right),
\end{aligned}
$$

and

$$
u=\left(\begin{array}{l}
m-3 \\
n-3
\end{array}\right)-3\left(\begin{array}{l}
m-4 \\
n-4
\end{array}\right)+\frac{3}{2}\left(\begin{array}{l}
m-5 \\
n-5
\end{array}\right)-\frac{1}{2}\left(\begin{array}{l}
m-6 \\
n-6
\end{array}\right)
$$


When $n=3$, the above reduces to

$$
\begin{aligned}
\tau_{i} & =\frac{2}{(m-2)(m-3)}\left(\sum_{\alpha ; i \varepsilon S} \bar{y}_{\alpha}-\left(\begin{array}{c}
m-1 \\
2
\end{array}\right) \tilde{y}\right), \\
\hat{\lambda}_{i j} & =\left\{\sum_{\alpha ; i, j \varepsilon S} \bar{y}_{S_{\alpha}}-(m-2) \tilde{y}-(m-3)\left(\hat{\tau}_{i}+\hat{\tau}_{j}\right)\right\} /(m-4), \\
\hat{\lambda}_{i j k} & =\sum_{\alpha ; i, j, k \varepsilon S} \bar{y}_{\alpha}-\tilde{y}-\hat{\tau}_{i}-\hat{\tau}_{j}-\hat{\tau}_{k}-\hat{\lambda}_{i j}-\hat{\lambda}_{i k}-\hat{\lambda}_{j k},
\end{aligned}
$$

where $\tilde{y}=6 \sum_{\alpha} \bar{y}_{S_{\alpha}} / \mathrm{m}(\mathrm{m}-1)(\mathrm{m}-2)$ is the overall mean of means.

If $\sigma^{2}$ is the variance of the experimental unit total response in blends of cultivars and if the replications are used for each blend in the orthogonal design

$$
\begin{aligned}
\operatorname{var}\left(\hat{\tau}_{i}-\hat{\tau}_{i^{\prime}}\right)=4 \sigma^{2} /\{r(m-2)(m-3)\}, & i \neq i^{\prime} \\
\operatorname{var}\left(\hat{\lambda}_{i j}-\hat{\lambda}_{i j^{\prime}}\right)=2(m-3) \sigma^{2} /\{r(m-2)(m-4)\}, & i \pm j^{\prime} \\
\operatorname{var}\left(\hat{\lambda}_{i j k}-\hat{\lambda}_{i j k^{\prime}}\right)=\frac{2 \sigma^{2}}{r}\left\{\frac{(m-5)^{2}}{(m-3)^{2}}+\frac{2(m-5)^{2}}{(m-3)^{2}(m-4)^{2}}+\frac{4}{(m-3)^{2}(m-4)^{2}}\right\}, & k \neq k^{\prime} .
\end{aligned}
$$

Expressions for the solutions of parameters can be similarly obtained for other designs of this type, when the interest is in drawing inferences about higher order specific mixing abilities. An analysis of variance can be obtained by standard methods.

\section{APPLICATIONS AND CONCLUDING REMARKS}

Several experiments of the type described above have been conducted and the above statistical analyses are considered to be appropriate for them. Three of these are described below. In the first one the experimenter, Steven Kauffka, Cornell University, was interested in biomass production of six species or cultivars in mixtures of three. There were 20 
such mixtures. His interest centered on general, first order, and second order mixing effects. The experiment design was a randomized complete block design. In a second experiment he used a balanced incomplete block design.

The models and analyses discussed here are not confined to intercropping experiments only. For a second application, preference ratings for eight soft drinks in mixtures (groups) of four using a doubly balanced incomplete block treatment design of 14 blocks has been conducted by Raghavarao and Wiley (1986). Their interest centered on first order specific mixing competing effects of one soft drink on another on preference ratings by individual users of soft drinks.

The results of this paper are also useful in studying the effects of one question on another in a survey design using the block total procedure of Raghavarao and Federer (1979). Many other applications could be made. Unequal numbers of cultivars or items in a mixture may also be used. D. B. Hall, described procedures for such situations in a 1975 Cornell University Masters Thesis entitled "Minimal designs to estimate bi-specific mixing ability." Note that solutions for $\tau_{i}=\delta_{i}+\tau_{i}^{*}$ were obtained here. To obtain solutions for $\delta_{i}$ and $\tau_{i}^{*}$ individually it is necessary to include uni-blends or monocultures.

\section{REFERENCES}

Raghavarao, D. and Federer, W. T. (1979). Block total response as an alternative to the randomized response method in surveys. J. Royal Statist. Soc., Series B, 41(1), 40-45.

Raghavarao, D. and Wiley, J. B. (1986). Testing competing effects among soft drink brands. In Statistical Design: Theory and Practice. Proceedings of a Conference in Honor of Walter T. Federer. Eds. C.E. McCulloch, S.J. Schwager, G. Casella, and S.R. Searle, pp. 161-176. Cornell University Press, Ithaca, New York. 